\title{
Enhancing the Building Information Modeling Lifecycle of Complex Structures with IoT: Phases, Capabilities and Use Cases
}

\author{
Larissa Gebken \\ University of Hamburg \\ gebken@informatik.uni- \\ hamburg.de
}

\author{
Prof. Dr. Paul Drews \\ Leuphana University of \\ Lüneburg \\ paul.drews@leuphana.de
}

\author{
Prof. Dr. Ingrid Schirmer \\ University of Hamburg \\ schirmer@informatik.uni- \\ hamburg.de
}

\begin{abstract}
In the construction sector, projects for building complex structures often exceed estimated costs and time. Therefore, enterprises in this sector rethink and improve their processes. For addressing this challenge, the discipline of Building Information Modeling (BIM) developed new process models to optimize the lifecycle of structures by leveraging technology and data sharing. Recently, enterprises explored the use of IoT technology for generating valid and up-to-date data. In this paper, we present an IoT capabilities map for BIM. This map employs BIM phases and capabilities to provide a structured overview of use cases from the literature and an empirical study we conducted in the rail construction sector. The map helps to guide further research in this area and provides blueprints for companies in the construction industry that seek to make use of IoT for improving their processes.
\end{abstract}

\section{Introduction}

In many industries, the digital transformation changes the existing ways of working. Likewise, the construction industry alters their processes by a new way of thinking called Building Information Modeling (BIM) to support the collaboration between the stakeholders. Especially for complex structures, companies involved in the process rely on a valid data and information basis. One possibility of improving the validity and timeliness of data is to use Internet of Things (IoT) technology. On their journey of IoT adoption, many companies are searching for and exploring IoT use cases. For this activity, companies have to be creative in order to rethink their processes. Furthermore, they also need necessary technological skills and experience. Currently, there is no systematic overview, which shows how IoT can be applied in the lifecycle of complex structures. Such an overview could be used by companies as a blueprint for identifying IoT use cases. For closing this gap of a missing overview, we address the following research question:

Which are use cases for applying IoT in the BIM lifecycle of complex structures and how can they be structured?

To answer this question, we start with a review of the literature related to the diffusion of IoT, BIM and the use of IoT in BIM. In the third section, we describe our design science-based research approach. In the results section, we present a three-layered map that employs BIM phases and capabilities to structure IoT use cases in the construction sector. Section five comprises results from an expert evaluation of the map. In section six, we discuss a generalization of the results and limitations. The paper closes with a conclusion.

\section{Related Research}

\subsection{Diffusion of Internet of Things (IoT) Technology}

IoT as an emerging technology already proved its applicability and potential impact in several industries [37]. Hence, many companies are already aware of IoT, but they are still in the process of identifying and realizing potential use cases. In the theory of diffusion of innovations, these activities (persuasion, decision and implementation) are part of the innovation-decision process [30]. These steps are known to be very timeconsuming and complex as it is necessary to have a deep understanding of both the business processes and the technology for successfully implementing a use case. In their innovation-decision process, companies could benefit from structured overviews of IoT use cases in their own and related industries as a source of inspiration for their own endeavors and for learning from the experiences of other companies.

IoT currently receives great attention as it allows to connect the physical and the digital world. IoT is a 
technology that enables its users to generate real-time data about the condition of a physical object like its location, movements or temperature. Such data can help to increase efficiency of existing processes and to develop new services [3]. So-called smart things or objects help to digitally perceive the environment and to initiate interaction [17]. These objects are called the "thing level" and comprise devices like sensors, actuators and RFID chips. A connection among these objects is realized via standardized networks such as the Internet [5], which is called the "internet level". In order to work purposefully with the collected data, it is necessary to add semantics or pattern recognition. This "semantic level" ensures an appropriate representation of the data [3].

\subsection{Building Information Modeling (BIM)}

$\mathrm{BIM}$ is rooted in the research area of Architecture, Engineering, and Construction (AEC) with a focus on building technology. So far, it has received only little attention from the IS community. The combination of AEC and IS research could offer new research opportunities as well as benefits for companies [24]. The main goal of BIM is to avoid the high cost and time overruns of construction projects as well as quality deficiencies [23].

BIM supports the collection and management of data throughout the lifecycle of a building and is defined as: "[...] a Business Process for generating and leveraging building data to design, construct and operate the building during its lifecycle. BIM allows all stakeholders to have access to the same information at the same time through interoperability between technology platforms." [26]

BIM demands for inter-organizational collaboration throughout all phases from planning and construction to operation [31]. Because buildings are usually planned, build and operated by many different companies, project work requires integrating strategy phases. Therefore, the requirements of the actors involved need to be documented for the Project Information Models (PIM) and Asset Information Model (AIM) [7, 8].

For our empirical study, we integrated three existing process models for BIM from the literature. As a starting point, we took the most comprehensive models PAS 1192-2:2013 and PAS 1192-3:2014 of the British Standard Institution [7, 8]. While PAS 1192-2:2013 focuses on the PIM strategy, planning and building phase [7], PAS 1192-3:2014 covers the AIM strategy and operational phase [8]. We also considered the approach of Borrmann et al. as it combines all BIM phases in one model and includes more detailed sub phases called service phases (SPH) for complex structures [6].

\subsection{IoT in BIM: State of the art}

Generating and leveraging the multi-dimensional information models pose a technical and organizational challenges due to the high number of participants and multifold requirements. If the participants in the BIM lifecycle are not constantly provided with relevant and updated data, there is a risk that information asymmetries occur. Therefore, the automatic acquisition and processing of data has a great potential for reducing workload and for avoiding errors [12]. One way to meet this challenge is to use IoT. In the following, we will describe the state of the art of research on using IoT in BIM.

Chen et al. took a literature-based approach to develop an integrated conceptual framework for bridging BIM with the real world. The framework summarizes types of data sources (laser scans, geo information systems, sensors, RFID, camera/video) that can be used and relates them to capabilities [12].

Alizadehsalehi and Yitmen demonstrate how wireless-sensor-networks and RFID chips could enrich existing digital models in the context of a university building [2]. Furthermore, they also grouped possible use cases into capabilities.

Several papers present specific use cases of integrating IoT into BIM. These are mainly stemming from the field of the construction of buildings. Niu et al. for example implemented a first prototype for integrating IoT into the digital information models of BIM for automating the monitoring of buildings [27]. Another use case was developed by Kim et al. [22]. To ensure the security of workers, they combined a realtime location system of workers and digital information models of BIM. Wang et al. integrated light detection and ranging into BIM to enable real-time checking of the light system and to improve the quality of the building [36].

The existing research shows, which capabilities for IoT related topics are interesting as well as distinct use cases of IoT application in the BIM context. Therefore, there is still a lack for a guiding structure, which provides an integrated overview of the BIM phases, capabilities and use cases for IoT in the lifecycle of complex structures.

\section{Research Approach}

We chose the design science paradigm to enable the combination of the methodological approach of research and problem solving in practice [20]. In order to achieve grounded findings, the design science process model according to Peffers et al. has been applied iteratively [28]. The first step was to identify the problem and the 
motivation to solve it. In this step, the existing literature was taken as a starting point for identifying the current state of the art and for framing the problem. We conducted a systematic keyword literature review according to vom Brocke et al. [35]. Main keywords were "Building Information Modeling", "Internet of Things" and "Capability". To extend the search, we also included IoT related topics like "RFID" and "sensor" and combined the keywords with different Boolean operators. We searched for publications in the AIS Basket of Journals, top four IS conferences and construction journals according to Chen et al. [12]. We also used ACM, Google Scholar, AIS eLibrary, JSTOR, ProQuest and Elsevier. Based on the results of this search, we derived capabilities and grouped the use cases.

Because the literature is mainly focusing on construction of buildings, we conducted an empirical study in the context of complex infrastructure undertakings in cooperation with a rail transport company and an IT consultancy based in Germany. Eight guideline-based interviews with nine experts in different roles were conducted. Two of the interviewees are project managers, one is a project agent, and four are project engineers and two work for IT service providers. In addition, we analyzed six documents from the participating companies, which extended the content of the interviews. These documents include BIM and IoT strategy plans as well as BIM project related documents. We transcribed the interviews and coded the transcripts and documents. We revised the codes iteratively and clustered them. The main results of the empirical study were the validation of the literature-based use cases and capabilities and the extension for rail-specific use cases.

To develop and design the IoT capabilities map for BIM, we first compared existing BIM frameworks. When we conducted this study, only national BIM frameworks existed. We compared the standards PAS1192-2 [7] and PAS1192-3 [8], COBie UK [1] and the Singapore BIM Guide Version 2 [9]. COBie UK is a framework that only covers the operational phase but not the entire lifecycle of a structure. Since the entire BIM lifecycle should be considered, COBie UK 2012 was not considered further. We assessed the BIM framework based on the definition of BIM, whether there is an existing reference process, fitting for infrastructure purposes and existing information requirements. As a result of the comparison, PAS11922 and PAS1192-3 are subsequently extended to an integrated lifecycle $[7,8]$. The transfer for the adjustment of the reference process for complex infrastructure projects is carried out with the help of the reference process according to Borrmann et al. [6].

We went through each use case and examined whether there is relevant information in the BIM frameworks, existing BIM good practice use cases and IoT integration requirements. We merged the results and mapped use cases and capabilities. In this paper, we only present the capability map. Due to limited space, we cannot present the use cases in detail.

We evaluated the IoT capabilities map for BIM with the aid of two guideline-based interviews with three experts to identify potentials for improvement.

\section{Results}

In the following chapter, we present the IoT capabilities map for BIM. It includes the BIM phases, capabilities and use cases (cf. Figure 1 and Table 1). This three-layered map provides an overview of how IoT can be implemented in BIM for supporting the planning, building and operation phases of a complex structure.

\subsection{Phases of the IoT capabilities map for BIM}

The IoT capabilities map for BIM is structured into five phases (cf. Figure 1):

1. Strategy phase-Project Information Model (PIM)

2. Planning phase

3. Building phase

4. Strategy phase-Asset Information Model (AIM)

5. Operational phase

In the strategy phase of the PIM, a cost-benefit analysis needs to be conducted. Furthermore, the employer's information requirements (EIR) have to be raised and a competence request of possible contractors is conducted. In addition, the contractors should show how the interaction between BIM and IoT is planned and document it in a BIM-IoT execution plan (BEIP) before and after closing the contract [7]. Depending on how the services are assigned, the strategy phase must be carried out several times in the planning and building phase because of a high division of labor in the complex infrastructure projects [6-8]. The planning phase and the building phase are separated in sub-phases. Depending on the use case, a distinction has been made between how the interaction of BIM and IoT can take place and which existing digital information models from the BIM area can be used (cf. section 4.2.). If it is planned to exploit the capability in the operational phase, the strategy phase-AIM must be examined. For the AIM, the cost-benefit analysis should be conducted again. For the transfer to the operational phase, it is important to check the EIR again. Some requirements might not be known at the beginning and it is necessary to prove which kind of information can be reused. To make this more concrete, the BEIP is necessary again 
$[7,8]$. Subsequently, in the operational phase, it needs to be inspected whether the respective use case could be used and how the interaction of BIM and IoT can take place. Again, this is a combination of BIM good practice use cases, requirements and the BIM-IoT use cases (cf. section 4.2.).

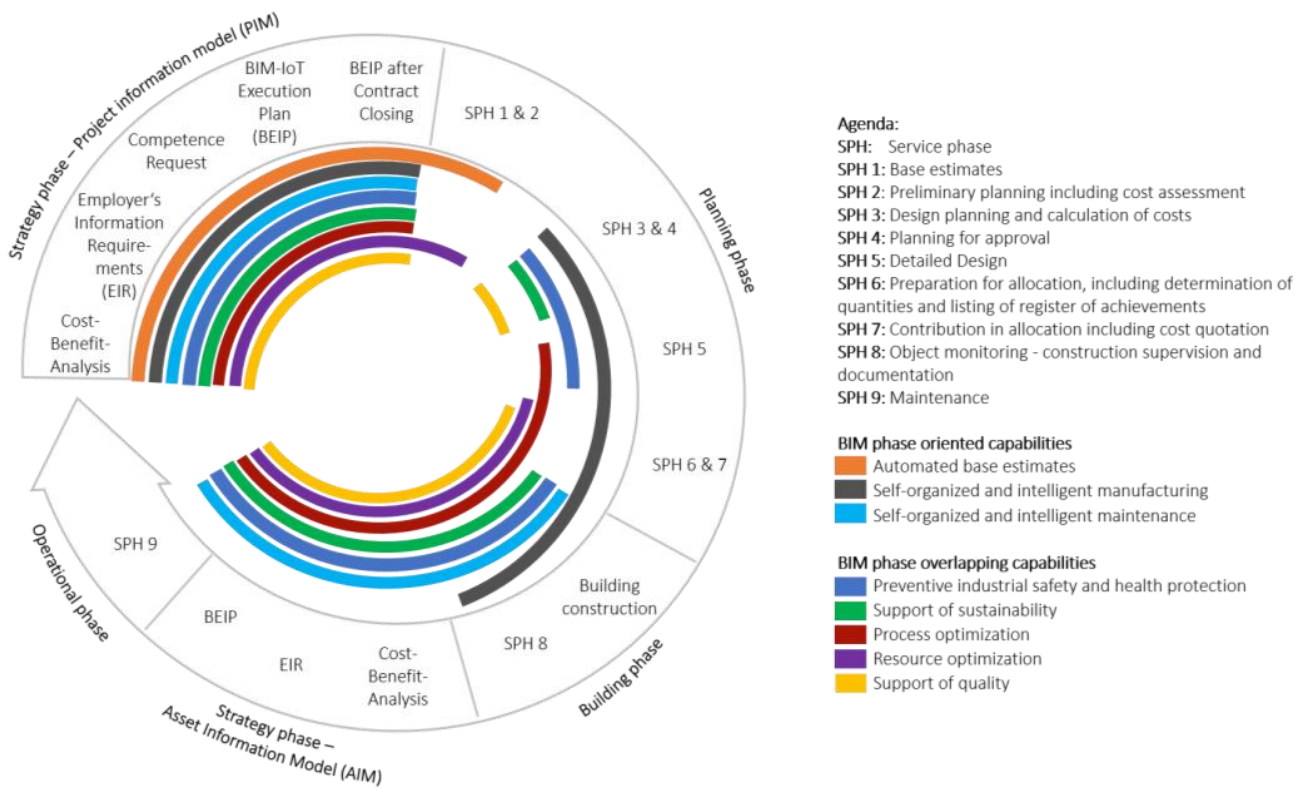

Figure 1. IoT Capabilities Map for BIM Based Upon [6-8]

\subsection{Capabilities of the IoT capabilities map for BIM}

The IoT capabilities can be grouped in BIM phaseoriented and overlapping capabilities (cf. Figure 1). While BIM phase-oriented capabilities include automated base estimates, self-organized and intelligent manufacturing and self-organized and intelligent maintenance, BIM phase overlapping capabilities include preventive industrial safety and health protection, support of sustainability, process optimization, resource optimization and support of quality [2, 12].

In case of establishing automated base estimates, companies are supported by a quicker base estimate and more detailed information. The focus of this capability is in the planning phase resp. in SPH 1 (base estimates) (cf. Figure 1, orange). In this phase, it is necessary to implement the thing, semantic and internet level. The results of stocktaking should be implemented as automated 3D inventory modelling. The critical examination is necessary in order to generate the benefit for the base estimates [D2, D3, I1, I6, I7].

The use of self-organized and intelligent manufacturing (cf. Figure 1, grey) can contribute to increase the quality and to avoid rapid wear of structures. The basis are 3D models of SPH 3 (design planning and calculation of costs) and the extension by cost and time components in SPH 5 (detailed design).
The realization of thing, semantic and internet level needs to be done in the construction phase. Subsequently, the results can be used in SPH 8 (object monitoring - construction supervision and documentation) [D2, D3, I1, I4, I6].

According to the experts, companies should focus on self-organized and intelligent maintenance (cf. Figure 1, cyan), since the operational phase of rail structures cost about $80 \%$ and the operation needs to be ensured over decades. When considering the use cases, there are two implementation options. Ideally, the requirements for the operational phase are collected in the strategy phasePIM. This enables implementing the thing level already in the construction phase. Since the precise formulation of the EIR is still a challenge, the EIR for operation should be raised in the strategy phase-AIM. In addition, it is necessary to examine which data and which things can be reused [7, 8]. If IoT has not been used, thing, semantic and internet level need to be implemented and the results integrated into the digital information models. To achieve benefits out of the related use cases, it should be checked if data can be reused and the results can be displayed in a dashboard [D2, D3, I1, I5-I7].

Within the capability of preventive industrial safety and health protection (cf. Figure 1, blue), IoT can support the long-term health of workers involved in construction or maintenance and thus to compliance with legal conditions. As the basis for integrating IoT data, 3D models of SHP 3 and 4 (planning for approval) 
or 4D models with time scopes in SPH 5 can be used. The interaction of BIM and IoT takes place in SHP 8 and the operational phase. All three levels of IoT need to be implemented and the results should be integrated in the models named before. In the strategy phase-AIM, companies should check whether the use cases are also interesting for the operational phase. The implementation of IoT or the check if reuse of data/IoT levels is possible needs to be done [D2-D4, I1, I3-I8].

IoT can also support engineers in the BIM lifecycle to enforce ecological sustainability (cf. Figure 1, green). Especially, complex rail structures are often struggling with a large number of critical stakeholders. The focus support of sustainability is primarily in the operational phase. As a part of the SPH 3, the corresponding 3D models have to be created for the subsequent enrichment with IoT data. The implementation of IoT takes place during the building phase. Therefore, it is necessary to prepare thing, semantic and internet level. The extracted IoT data can be used to enrich 3D models of SPH 3 and 4 or SPH 8 (object monitoring - construction supervision and documentation). It is necessary to examine to what extent the IoT data can be used to implement long-term ecological sustainability support for complex structures [D2-D5, I1, I3, I4, I6-I8].

Process optimization (cf. Figure 1, red) is one of the most promising capabilities for IoT, because delays could be decreased and the time and cost spending could be reduced. Furthermore, decisions can be made on a valid data base. Anyway, it is important to focus on critical processes to ensure a good cost-benefit-ratio. The focus of the process optimization is in the building phase. 4 and 5D models of SHP 5 and SHP 7 (contribution in allocation including cost quotation) have to be enriched by IoT data. Therefore, the implementation of thing, semantic and internet level is necessary to enable self-disclosure about time and costs. For example, the construction progress control or the earned-value analysis on the exact day-end date can be enriched with real-time information. Since the use cases are thematically related, the mapping within a dashboard is recommended. This enables the reuse of IoT data. Depending on the capability, it is necessary to check whether the implementation is relevant for the operational phase in the strategy phase-AIM. Hence, it is important to check if data or level reuse is possible [D2-D4, I1, I3-I7].

An efficient resource usage is also important for the construction of complex structures (cf. Figure 1, purple). For resource optimization, the attention must be paid to the construction and operational phases. There are still no BIM use cases in the planning phase, which deal exclusively with resource optimization. If the resource optimization is targeted, thing, semantic and internet layer should be designed. This has to take place during construction or operational phase depending on the time of use. Corresponding dashboards have to be developed which represent the desired information. The networking of the respective optimization measures should also be considered. In the context of the strategy phase-AIM, it has to be examined whether and to what extent the existing data can be used or which levels can be reused. If the use case has not been realized previously, the implementation needs to be caught up [D2, I1, I5-I7].

The interaction of BIM and IoT is also shown for the support of quality (cf. Figure 1, yellow) in the capability map. The 3D models of SPH 3 and 4 which focus on quality could be enriched by IoT data. During the building phase, the three levels of IoT should be established. The linkage with the 3D inventory models of the construction site documentation should be used to be able to monitor the quality on IoT-based data. For the transition to the operational phase, it is necessary to examine whether and what data and things are of interest to the operators. Thus, an adjustment is necessary. If the use case has not been realized, this needs to be fetched up after the strategy phase-AIM in order to enable the implementation of BIM-based maintenance and repair measures including the IoT-generated data [D2, D3, I1, I4-I8].

\subsection{Use cases of the IoT capabilities map for BIM}

As mentioned in sections 4.1 and 4.2, the IoT use cases were matched to the capabilities. Overall, 35 capabilities could be identified. Therefore, the 14 use cases from literature where validated and extended by the qualitative study. Table 1 summarizes all use cases. The sources from literature are illustrated with numbers, the sources from the qualitative study are marked with an "I" (Interviewee) and "D" (Document) and consecutive numbers. In the following, we will only highlight specialties of the use cases.

In the qualitative study, we found out that for the category automated base estimates (cf. Table 1, orange) sensors should be used for special buildings. Zhang and Bai also show that there is a need for new technologies and that technologies like laser scanners are outdated [38]. For the base estimate of simple lines, other, cheaper technologies like using drones should be used. The first pilot phase already started. Because of the implementation costs, it is important that stakeholders consider the cost-benefit ratio for this use case [I6, I8].

For the capability self-organized and intelligent manufacturing (cf. Table 1, grey) three use cases were identified. Repetitive tasks in case of the railway sectors could be digging pits or mounting of screws [I1, I3, I4, 
I6, I-9]. The authors reduced the error-proneness of building components by using RFID chips to increase the comprehension of manufacturing [4].

As mentioned in section 4.2., self-organized and intelligent maintenance (cf. Table 1, cyan) is one of the categories companies should focus on when adopting IoT [32]. The automated monitoring of buildings is a relevant use case in this area. Moon et al. implemented a first prototype for integrating IoT into the digital information models of BIM [25]. In addition, there are use cases that show how to automate the repair process in case of emerging errors $[34,36]$ or how to adjust the maintenance intervals based on automated equipmentbehavior-learning [21]. An IoT solution for the continuous monitoring of lines with regular trains or trains for measuring already exist, but the results are not used in the BIM lifecycle yet [18]. The experts already identified critical elements for the railway infrastructure. This could be railroad switches, gas pipelines, and statics of the respective structures, axle counters and signals. Especially, the railroad switches are one of the key elements of a railway infrastructure and a failure leads to long delays in the operational phase. Predictive maintenance is seen as the most advanced use case. The condition of structures are permanently monitored and infrastructure components are monitored and diagnosed. In the end, this can ensure that suppression is accelerated, wear and tear avoided, and predictions about upcoming disruptions can be made. First applications for railroad switches, switch heaters and railroad crossings are already planned. The use cases are partly based on each other and can be combined differently [D1, I1-I8].

Preventive industrial safety and health protection (cf. Table 1, blue) with IoT can support companies by carrying out regulations in the whole BIM lifecycle. Additionally, it can avoid high risks of death. There are already pragmatic solutions for control of pollutant leakage and it is questionable whether the operation with IoT is a gain for safety. Qian and Lin developed a scenario to reduce safety risks on the construction field [29] and Ding et al. used motion patterns of workers to evolve an early warning system in tunnels [15]. Other authors used motion patterns to categorize safety areas $[22,27]$. The usage of motion patterns of workers is not allowed in some countries. Therefore, possible capabilities in this field were not included. The experts questioned if real time monitoring leads to a big increase of safety. [I1, I3, I6, I7, I8].

The support of sustainability is divided in three use cases (cf. Table 1, green). One of the main fields in this area is the continuous control of energy [10]. Another way of supporting sustainability is to do recycling. By doing a health check of the materials, the reusability can be supported by BIM and RFID chips [33]. Since most infrastructure constructions are not built on the green field but on already existing routes and elements, the reusability test is an interesting use case. However, it must be checked if an adaptation is legal [I1, I3, I5-I8].

Process optimization use cases were also found in the research process (cf. Table 1, red). Especially, the automated billing can replace existing billing processes for complex infrastructure projects. Currently, the companies use internal standards. These are too extensive to fill them fully and correctly without technical assistance. The automated update of the construction progress is critical due to an unclear accuracy. Many different factors have to be taken into account at the construction site and the experts think that monitoring them completely with digital information models of the BIM and IoT is unrealistic. As the delay in construction can cause major financial losses it is one of the biggest risks for the employer or contractor. In order to prevent such losses, a framework has been developed in the BIM context, which should help to assess the project risks and that could make use of IoT [11]. In order to measure the work progress of construction tasks, the integration of BIM and IoT has been undertaken [14]. The additional assessment of experts is nevertheless necessary. The optimization of the construction site is also of interest. Griva et al. used RFID chips to optimize the construction site logistics [19]. In addition, the automation of repair processes is a field of interest. Another use case is the identification of new projects. The data collection did not reveal how complex infrastructure projects are usually created. By using BIM and IoT, it is possible to start projects on demand based on a valid database [I1-I3, I5-I8].

In the field of resource optimization (cf. Table 1, purple), the optimization of building machine use is one of the most promising use cases. In addition, costs can be saved through the automated testing of whether rail locos can be used in times of non-use for construction site logistics. The locomotive is one of the most expensive resources in the railway. Therefore, it should be subject to optimization. By using the generative design based on a large amount of data and various algorithms, it is possible to calculate the optimal construction of a structure. In addition, the use of automated feedback loops makes it possible to learn from the behavior of structures and to gain insights for further construction projects or to use the collected information within the framework of the operation. Construction materials like carbon fiber could be used to enable self-disclosure of materials [I1, I3, I6-I8]. While in one use case RFID chips monitor the degree of wear on resources [13], another uses RFID chips and GPS data for automated theft detection [14]. 
Table 1. Use Cases of the loT Capabilities Map for BIM

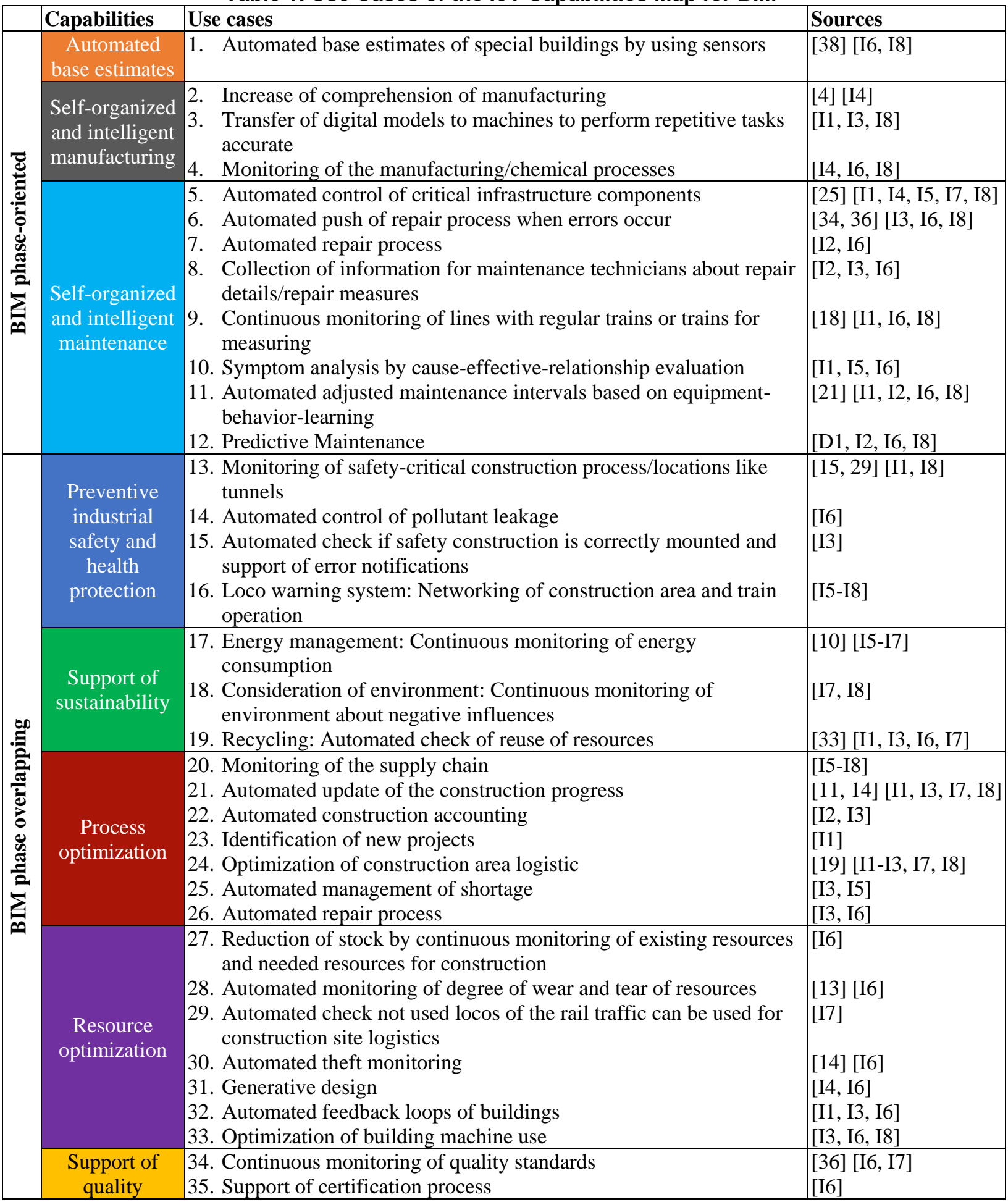

Also in the category support of quality, (cf. Table 1, yellow) experts mentioned additional capabilities. To achieve the support of quality standards of structures,
IoT has already been used. Real-time monitoring of quality standards based on sensors (e.g. pressure sensors) has been implemented [36]. It stills needs to be 
identified, which quality standards and certification processes are important in case of rail transport [I6-7].

\section{Evaluation}

Overall, the qualitative evaluation by experts showed that the structure of the capability map has been chosen appropriately, the use cases are clearly illustrated and the capabilities summarize all relevant use cases known so far. According to the experts, the 35 use cases show realistic scenarios and the capabilities provide a useful structure. For the future, it is important to continuously check whether there are new use cases due to new technologies or process innovation. In the evaluation, no further capabilities and use cases were mentioned. For the implementation of the use cases, it is necessary to examine which algorithms and things should be used. The IoT capabilities map for BIM can be used as a basis for discussions in the BIM committees to push the diffusion of IoT. The evaluation confirmed that the map is interesting for employers and contractors of complex infrastructure projects. Primarily, it is suitable for promoting the diffusion of innovation in the railway and civil engineering sectors. It serves as a basis to extend the current BIM use cases and draw attention from politics to IoT topics. Thus, in the best case, it will be possible to gain financial support for piloting one of the use cases. To enable the implementation of IoT, it is necessary to do a lot of persuasion in the committees. In particular, because BIM is already a challenge and the degree of maturity in complex infrastructure projects is not high enough, yet. Companies should not only implement one use case after another but they should ensure to realize synergy effects by integrating the planning of use case implementation. The IoT generated data should be used for several capabilities. Therefore, the related capabilities and use cases should be linked in a dashboard. Overall, the BIM frameworks are not detailed enough thus leading to high efforts in adopting it in a company. This also applies for the IoT capabilities map for BIM. Both approaches require that employers clearly formulate which information they exactly need. The experts also expressed further extensions for the integrated IoT capabilities map for BIM [E1, E2]:

- For each use case, algorithms and things should be listed for implementation.

- For each use case, it should be highlighted when the benefit arises.

- For each use case, recommendations for action or priorities should be extended.

- For each capability, possible extensions by other technologies (e.g. artificial intelligence) should explored.
Beyond the evaluation of the capabilities map, the experts also covered further contextual conditions that influence the adoption of BIM and IoT in the construction industry. First, one of the main challenges is that introducing BIM is still considered as an innovation for many companies and a field in which they lack required knowledge while they suffer from high pressure on the infrastructure projects at the same time. This leads to a lack of collaboration between stakeholders and the existence of silos so that the benefits of BIM cannot be realized. The non-adoption of BIM is expected to have a negative influence on IoT adoption. Second, the field is still facing major technical issues. The possibilities for visualizing special multidimensional information models like long haul routes are still missing. In addition, the extensive computing power, which is needed for IoT and the digital information models, can harm innovations. Third, for the construction sector, many laws and standards as well as historical grown structures and the long lifetime of buildings have to be taken into account as they might also slow down the adoption of BIM and IoT.

\section{Discussion}

In this paper, we focused on the research gap of a missing systematic overview of how IoT can be used in the BIM lifecycle of complex structures. By drawing upon existing literature and standards as well as on results from a qualitative-empirical study, we developed an IoT capabilities map for BIM which comprises 35 use cases. The evaluation of this map helped to identify some limitations as presented in the previous section. Furthermore, the results have not yet been related to the ISO 29481-1 (standard for BIM) as it was published after we conducted our study.

While our study focuses on the construction sector, some of the results might be useful for other industries as well. The planning, building and operation of other complex physical objects like cars or airplanes might benefit from the IoT use cases and capabilities we identified in the construction sector. The approach taken in this paper could be used as a blueprint for identifying potential areas of applying IoT and for supporting the decision making across the lifecycle. However, additional research is necessary for identifying the industry-specific characteristics of applying IoT in other industries and relate the findings to the map developed in this paper. For example, the use case "transfer of digital models to machines to perform repetitive tasks accurate" could be one of the most interesting for the automobile industry. 


\section{Conclusion and Outlook}

In this paper, we applied a design science process to develop a three-layered map that structures the use cases for IoT in the construction sector by drawing on the BIM approach. The evaluation showed that experts value the contribution of the capabilities map while they also demand future extensions regarding more detailed information about the technology applied in the use cases. They also highlighted that the progress of BIM adoption might not be advanced enough to broadly adopt IoT in the construction industry. Also, a lack of collaboration hinders the actors from improving the information management on the ecosystem level.

Future research and practitioners could use the map as a starting point for further exploring the use cases on a detailed level. Step-by-step, the structure of the map could be enhanced while also additional information on the cases could be made available in order to support the diffusion of IoT. Studies in related industries like car or airplane manufacturing could draw upon the structure presented in the paper while also considering industryspecific use cases and capabilities.

The digital transformation in the lifecycle of complex structures remains a complex and long-term endeavor. Other studies already showed that a missing big picture of the complex processes and the according business ecosystem architecture can lead to failure [16]. Therefore, we will further investigate if enterprise or business ecosystem architecture management can support the diffusion of innovations and digital transformation.

\section{References}

[1] AEC 3, "Required Information for Facility Operation. COBie-UK", July 2012.

[2] Alizadehsalehi, S. and I. Yitmen, "The Impact of Field Data Capturing Technologies on Automated Construction Project Progress Monitoring", Procedia Engineering, 161, 2016, pp. 97-103.

[3] Atzori, L., A. Iera, and G. Morabito, "The Internet of Things: A survey", Computer Networks, 54(15), 2010, pp. 2787-2805.

[4] Baars, H., D. Gille, and J. Strüker, "Evaluation of RFID applications for logistics: A framework for identifying, forecasting and assessing benefits", European Journal of Information Systems, 18(6), 2009, pp. 578-591.

[5] Bassi, A., H. Europe, and G. Horn, "Internet of Things in 2020: A Roadmap for the Future", Information Society, European Technology Platform on Smart Systems Integration, 2008.
[6] Borrmann, A., H. Bramann, W. Eckart, M. Herrscher, J. Koggelmann, M. König, T. Liebich, I. May, D. Mondino, H.G. Oltmanns, and J. Tulke, Konzept zur schrittweisen Einführung moderner, IT-gestützter Prozesse und Technologien bei Planung, Bau und Betrieb von Bauwerken: Stufenplan zur Einführung von BIM, planen bauen 4.0, 31.12.2015.

[7] British Standards Institution, "Specification for information management for the capital/delivery phase of construction projects using building information modelling. PAS 1192-2", 91.010.01, February 2013.

[8] British Standards Institution, "Specification for information management for the operational phase of assets using building information modelling. PAS 1192-3", 91.010.01, March 2014.

[9] Building and Construction Authority, "Singapore BIM Guide Version 2", Singapore, August 2013.

[10] Casini, M., "Designing the third millennium's buildings", in Smart Buildings: Advanced Materials and nanotechnology to improve energy-efficiency and environmental performance, M. Casini, Editor. 2016. Elsevier Verlag: Amsterdam.

[11] Chang, C.-Y., "Risk-bearing capacity as a new dimension to the analysis of project governance", International Journal of Project Management, 33(6), 2015, pp. 1195-1205.

[12] Chen, K., W. Lu, Y. Peng, S. Rowlinson, and G.Q. Huang, "Bridging BIM and building: From a literature review to an integrated conceptual framework", International Journal of Project Management, 33(6), 2015, pp. 1405-1416.

[13] Costin, A., N. Pradhananga, J. Teizer, and E. Marks, "Real-Time Resource Location Tracking in Building Information Models (BIM)", in Cooperative design, visualization, and engineering, Y. Luo, Editor, Osaka, Japan, September. 2012. Springer: Berlin.

[14] Dave, B., S. Kubler, K. Främling, and L. Koskela, "Opportunities for enhanced lean construction management using Internet of Things standards", Automation in Construction, 61, 2016, pp. 86-97.

[15] Ding, L.Y., C. Zhou, Q.X. Deng, H.B. Luo, X.W. Ye, Y.Q. Ni, and P. Guo, "Real-time safety early warning system for cross passage construction in Yangtze Riverbed Metro Tunnel based on the internet of things", Automation in Construction, 36, 2013, pp. 25-37.

[16] Drews, P. and I. Schirmer, "From Enterprise Architecture to Business Ecosystem Architecture: Stages and Challenges for Extending Architectures beyond Organizational Boundaries", in 2014 IEEE 18th International Enterprise Distributed Object Computing Conference workshops and demonstrations (EDOCW): 1-2 September 2014, Ulm, Germany, G. Grossmann, Editor, Ulm, Germany. 2014.

[17] Giusto, D., L. Atzori, A. Iera, and G. Morabito, eds., The Internet of Things: 20th Tyrrhenian Workshop on Digital Communications, Springer Verlag, New York, USA, 2010. 
[18] Goodman, D.L., J. Hofmeister, and R. Wagoner, "Advanced diagnostics and anomaly detection for railroad safety applications: Using a wireless, IoT-enabled measurement system", in 2015 IEEE AUTOTESTCON, National Harbor, USA. 2015.

[19] Griva, A., C. Bardaki, K. Pramatari, and D. Georgios, "Mapping Moving Object Events into a Network of Object Flows to Support Decissions", in Proceedings of European Conference on Information Systems. 2016.

[20] Hevner, A.R. von, S.T. March, J. Park, and S. Ram, "Design science in information systems research", MIS quarterly, 28(1), 2004, pp. 75-105.

[21] Jeong, S., J. Byun, D. Kim, H. Sohn, I.H. Bae, and K.H. Law, "A data management infrastructure for bridge monitoring", in SPIE. Smart Structures and Materials + Nondestructive Evaluation and Health Monitoring, J.P. Lynch, Editor, San Diego, USA. 2015.

[22] Kim, H., H.-S. Lee, M. Park, B. Chung, and S. Hwang, "Automated hazardous area identification using laborers' actual and optimal routes", Automation in Construction, 65, 2016, pp. 21-32.

[23] Latiffi, A.A., J. Brahim, and M.S. Fathi, "Roles and Responsibilities of Construction Players in Projects Using Building Information Modeling (BIM)", in Product lifecycle management in the era of internet of things: 12th IFIP WG 5.1 International Conference, PLM 2015, Doha, Qatar, October 19-21, 2015 : revised selected papers, A. Bouras, B. Eynard, S. Foufou, and K.-D. Thoben, Editors. 2016. Springer Verlag.

[24] Merschbrock, C. and B.E. Munkvold, "A Research Review on Building Information Modeling in Construction An Area Ripe for IS Research", Communications of the Association for Information Systems, 31(10), 2012, pp. 208 228.

[25] Moon, H.-s., H.-s. Kim, S.-y. Moon, H.-w. Kim, and L.s. Kang, "Prototype model of wireless maintenance operation system for bridge facility using VR and ubiquitous technology", in Proceedings of the 11th ACM SIGGRAPH International Conference, D. Thalmann, E. Wu, and Z. Pan, Editors.

[26] National Institute of Building Sciences, National BIM Standard-United States Version 3, buildingSMARTalliance ${ }^{\circledR}$, 2015.

[27] Niu, Y., W. Lu, K. Chen, G.G. Huang, and C. Anumba, "Smart Construction Objects", Journal of Computing in Civil Engineering, 30(4), 2016.

[28] Peffers, K., T. Tuunanen, M.A. Rothenberger, and S. Chatterjee, "A design science research methodology for information systems research", Journal of management information systems, 24(3), 2007, pp. 45-77.

[29] Qian, Q. and P. Lin, "Safety risk management of underground engineering in China: Progress, challenges and strategies", Journal of Rock Mechanics and Geotechnical Engineering, 8(4), 2016, pp. 423-442.

[30] Rogers, E., "Diffusion of Innovations", Free Press, 5th ed., 2003.

[31] Shen, W., Q. Hao, H. Mak, J. Neelamkavil, H. Xie, J. Dickinson, R. Thomas, A. Pardasani, and H. Xue, "Systems integration and collaboration in architecture, engineering, construction, and facilities management: A review", Advanced Engineering Informatics, 24(2), 2010, pp. 196-207.

[32] Soga, K., "Innovation in Instrumentation, Monitoring, and Condition Assessment of Infrastructure", in Multi-hazard Approaches to Civil Infrastructure Engineering, P. Gardoni and J.M. LaFave, Editors. 2016. Springer Verlag.

[33] Swift, J., D. Ness, N. Chileshe, K. Xing, and J. Gelder, "Enabling the Reuse of Building Components: A Dialogue between the Virtual and Physical Worlds", in Proceedings of the Unmaking Waste Conference. 2015.

[34] Volkov, A.A. and E.I. Batov, "Dynamic Extension of Building Information Model for "Smart" Buildings", Procedia Engineering, 111, 2015, pp. 849-852.

[35] Vom Brocke, J., A. Simons, B. Niehaves, K. Riemer, R. Plattfaut, A. Cleven, and others, "Reconstructing the giant: On the importance of rigour in documenting the literature search process", in Proceedings of the 17th European Conference on Information Systems, Verona. 2009.

[36] Wang, J., W. Sun, W. Shou, X. Wang, C. Wu, H.-Y. Chong, Y. Liu, and C. Sun, "Integrating BIM and LiDAR for Real-Time Construction Quality Control", Journal of Intelligent \& Robotic Systems, 79(3-4), 2015, pp. 417-432.

[37] Xu, L.D., W. He, and S. Li, "Internet of Things in Industries: A Survey", IEEE Transactions on Industrial Informatics, 10(4), 2014, pp. 2233-2243.

[38] Zhang, Y. and L. Bai, "Rapid structural condition assessment using radio frequency identification (RFID) based wireless strain sensor", Automation in Construction, 54, 2015, pp. 1-11. 